\title{
Senior Managers' Information Behavior in Current Emerging Ubiquitous and Intelligent Computing Environment
}

\author{
Vincent K Ong \\ Regent's University London \\ ongv@regents.ac.uk
}

\author{
Yanqing Duan \\ University of Bedfordshire \\ yanqing.duan@,beds.ac.uk
}

\author{
Mark Xu \\ University of Portsmouth \\ mark.xu@port.ac.uk
}

\begin{abstract}
Emerging ubiquitous and intelligent information systems, such as the Internet, social computing technologies and artificial intelligence (AI), have facilitated the increasing complexity and dynamism of operational and strategic information in a highly distributed environment. As a result, organizations have been busy seeking approaches and tools to support senior managers in coping with this challenge, from organizational learning to knowledge management, from competitive intelligence to business intelligence, and from management information systems to strategic (executive) information systems. Before embarking on formulating and developing these approaches and tools, senior managers' informational roles and information behavior should be understood. This paper explores factors influencing and shaping existing senior managers' information behavior in order to shed light on value-added approaches or technological solutions for supporting and improving informational roles of senior managers. The findings show that information behavior of senior managers is influenced and shaped by a number of factors, mainly the organizational actors and organizational situations, followed by their affective responses and the use of technological tools.
\end{abstract}

\section{Introduction}

Much has seemingly changed over the past three decades in the internal and external business environment, but Mintzberg's descriptions of managerial roles have not changed much. Information is still central to the performance of managerial roles. Senior managers are still functioning as the "nerve center" for the creation, collection and dissemination of information [1, 15]. However, the existing business environment is becoming more volatile and competitive. Emerging ubiquitous and intelligent information systems, such as the Internet, social computing technologies and artificial intelligence (AI), have facilitated the increasing complexity and dynamism of operational and strategic information in a highly distributed environment. As a result, organizations have been busy seeking approaches to support senior managers in coping with this challenge, from organizational learning to knowledge management, from competitive intelligence to business intelligence, and from management information systems to strategic (executive) information systems. The question is, before embarking on formulating and developing these approaches, what factors affect the processing of strategic information and do organizations know how the existing senior managers collect and process information for decision-making and problem solving.

The challenge is senior managers do not conform to formal information behavior or decision making models, instead, they are sporadically managing a mess, muddling through information as the business environment releases all kinds of signals and messages. Managerial works are still characterized by brevity, variety and discontinuity $[1,15]$. It is, therefore, not feasible to identify exact patterns of senior manager's information behavior. Prior research of information behavior suggests that information behavior is shaped by multiple factors, such as the individual's work situations and organizational contexts (situational factors), the affective feelings of individuals (affective factors), the thinking efforts of individuals (cognitive factors), and the systematic stages of process (systematic factors) [5, 6, 10, 11, 21, 25]. Hence, this study aims to explore factors influencing and shaping existing senior managers' information behavior in light of their informational roles and current competitive business environment. This may shed light on the knowledge for formulating and developing valueadded approaches for supporting and improving informational roles of senior managers. 


\section{Informational Roles of Senior Managers}

Mintzberg (1973)'s descriptions of managerial roles have not changed much even though the business environment has become increasingly complex and dynamic [1, 15]. Mintzberg identified three categories of managerial roles (interpersonal, informational, and decisional) that are critical for managing their managerial activities. The interpersonal roles (i.e. figurehead, leader, liaison) that derive from the manager's formal authority and unique position give rise to the informational roles (i.e. monitor, disseminator, spokesman), and in turn enable manager to perform his decisional roles (i.e. entrepreneur, disturbance handler, resource allocator, negotiator). The informational roles are manager's key roles that tie all managerial work together, connecting the interpersonal roles with the decisional roles. The informational roles, therefore, allow manager to function as the "nerve center" for the creation, collection and dissemination of information $[1,15]$, and to be effective in ubiquitous decision-making and problem solving. Thus, information behavior is central to the performance of managerial roles.

The informational roles of senior managers are becoming more challenging as the amount of operational and strategic information, from both the internal and external environment, is becoming more complex and dynamic. The advent of the Internet also provides the impetus to reexamine the informational roles of senior managers. Today, senior managers are facing more heterogeneous and geographically distributed information sources. It is becoming more difficult to monitor and scan the business environment. At the same time, senior managers are constantly under pressure to seek trigger, speculative and current information in order to process and disseminate information quickly to stakeholders. As a result, senior managers are facing challenges as strategy makers because they need evidence-based information to make decision and to plan and identify problems and opportunities.

Kotter (1999) found that the effective senior managers tend to rely actively on agenda setting and network building in order to tackle those challenges [12]. Senior managers are expected to use their informational roles to develop, connect and complete their agendas, and to build networks. As a result, senior managers spend most of their time with people, actively involved in a wide range of discussion topics (often short and disjointed conversations), skillfully ask questions and seek out information for making decisions and plans, as well as identifying opportunities and threats.

\section{Information Behavior}

From the information science perspective, information behavior is "those activities a person may engage in when identifying his or her own needs of information, searching for such information in any way, and using or transferring that information" [24, p.249]. Bawden and Robinson (2011) stated that information behaviour is inherently individual [2]. The focus is on individual human information related behaviors, including information seeking, information gathering and information manipulating activities. It comprises the process in which the individual searches for information in order to change his or her state of knowledge. During this process, the individual identifies and selects information sources; articulates a query, question or topic; extracts the information; evaluates the information retrieved; filters the irrelevant information, and interprets the information. The individual might go back and repeat the processes till his information needs are met. From the psychology perspective, information behavior focuses on the behavioral aspects of human and information behavior. Spink (2010) argues that information behavior is evolutionary, influenced and shaped by the cognitive, affective and societal development of individual [21]. Hence, information behavior includes the affective and socio-cognitive ability of humans in information processing. Empirical studies suggest that information behavior is shaped by multiple factors, such as the individual's work situations and organizational contexts $[8,22]$, the affective feelings of individuals [4, $13,19]$, and the thinking efforts of individuals [10, 26]. Choo's (1998) general model of information use indicates that individual information behavior is influenced and shaped by situational, affective and cognitive dimensions [6, 7] (see Figure 1).

Choo (1998) categorized the individual's work situations and organizational contexts as the situational dimensions based on Taylor's (1991) proposal of information use environments (IUEs) [23]. The thinking efforts (cognitive) of individuals shape information behavior by attempting to find information in order to bridge the situation gaps (cognitive gaps) when he or she recognizes an inability to act or understand a situation because of a lack of information $[6,8,26]$. The way individual perceives a gap is a good indicator of how the individual will go about bridging the gap and wanting the help [6]. The affective responses or feelings influence information behavior by channeling attention to certain issues or information, pointing out doubt and uncertainty, indicating likes and dislikes, and motivating effort [4, 6, 19]. For instance, information gathering is influenced by the user's mood or attitude towards the 
search task. A user in a mood that eagers for new ideas would tend to explore further, whereas a user who feels having enough information would tend to cease exploration quickly [11]. Kuhlthau's (1993) affectiveoriented model describes that the affective responses could ease the uncertainty, increase confidence in the course of information search and motivate individual's information processing experience [13]. For example, as the information search progresses, feelings will shift toward increased confidence and satisfaction if the search has been successful.

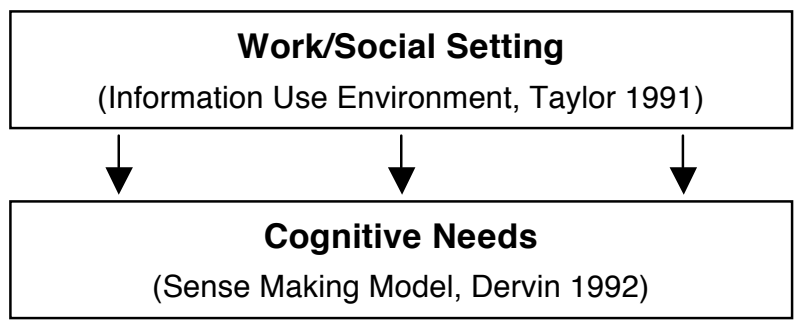

\section{Affective Responses}

(Information Search Process, Kuhlthau 1993)

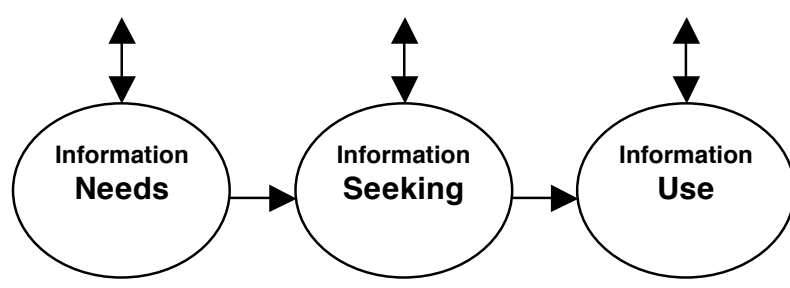

Figure 1. Model of Information Processing Behavior (adapted from Choo 1998)

Choo's (1998) general model of information use is an attempt to identify the major elements that influence the information behavior from both information science and evolutionary psychology perspective [6]. The model posits that information needs arise when an individual recognizes situations or problems in his or her information environment. This creates information gaps, which may lead to various emotion and responses, such as uncertainty, confusion and anxiety. As a result, the individual will intentionally and strategically seek information and solutions in order to bridge the information gaps. The individual then selects and processes information in order to understand a situation or solve a problem, leading to new emotion and responses, such as confidence, clarity and relief. However, if information seeking and use are unable to produce meaningful and relevant information or solutions, it can lead to negative emotion and responses.

Although many information behavior studies have been conducted $[2,5,9,13,24,25]$, information behavior of senior managers is still a gap that requires more understanding. It is increasingly important to understand and consider different factors that shape and influence senior manager's information behavior in current information age. If information behavior were merely systematic stages of process activities, it would be much easier to program those activities with computer-based information systems. However, it is a complex process that involves the situational, affective and cognitive dimension of individuals. The situational dimension is more likely to be identified and understood, but the affective and cognitive dimension will be much harder to identify and understand. The way human think and learn in complex situations are still mysterious for information systems designers to design and develop mechanisms to cope with these issues. However, it does not mean that researchers can ignore this aspect of study. Current research in cognitive and affective computing begins to study user's thinking, affect and emotion in information behavior $[4,22,26]$. An affective support in information processing activities will help reduce uncertainty and increase confidence, thus increase user's ability to construct meaning or make sense of the information [6]. This study will, therefore, potentially add knowledge to Choo's model of information processing behavior as the focus of this study is on senior manages' information environment and information processing.

\section{Methodology for the Study}

The aim of this study is to understand the existing information environment and information behavior of senior managers, and to explore factors influencing and shaping existing senior managers' information behavior. A qualitative and interpretive approach was adapted to induce answers or issues (themes) raised by senior managers. Semi-structured interview was employed in the study because a semi-structured interview combines a structured agenda with the flexibility to ask and probe subsequent questions [20]. In one-to-one semi-structured interview, open-ended questions provide senior managers with the sense of control, as well as the possibility for senior managers to elucidate his or her answer or introduce further arising information, ideas and issues.

Twenty-five senior managers were selected using a purposeful sampling strategy. The sample was taken from the Financial Analysis Made Easy (FAME) online database with the Standard Industrial Classification (SIC) code of 65 - Financial Intermediation (except Insurance and Pension Funding); code 66 - Insurance and Pension Funding 
(except Compulsory Social Security); code 67 Financial Auxiliary; code 63 - Travel; code 70 - Real Estate. These senior managers are key informants as they were selected from information intensive industries, such as financial industry, insurance industry and real estate industry, and all of them are involved in strategic decisions activities (80\% of Senior Executive, 20\% of Middle Executive). Most of the participants worked in medium and large organizations in the City of London, the financial hub of the UK. Their job titles include Vice President, Chief Executive Officer, Chief Operating Officer, Chief Finance Officer, Managing Director, Deputy Managing Director, Service Director, Operations \& Systems Director, Trading Director, Head of CEO, Head of IT Strategy, Head of Corporate Governance, Strategic Planning Manager, Customer Centre Manager and Business Development Director. Each interview lasted about 45 to 75 minutes and was conducted in the workplace of the interviewee. All interviews were digitally recorded and transcribed verbatim. The thematic qualitative analysis (TQA) method (Mason 2002) was employed for inductive analysis [14]. Firstly, generalized themes were elicited from the raw data and organized into meaningful themes in first and second order. In total, 22 first order themes were elicited, followed 8 second order themes. Secondly, a detailed interpretive conceptual analysis and mapping was conducted to interpret the meanings of themes and to identify any consensus, dilemmas, and contradictions by reading and re-reading quotes from each emerging themes [17]. Interpretation reliability was assessed through inter-rater reliability analysis [16], in which two research colleagues performed double blind interpretation with a view to reaching the inter-subjective agreement among interpreters. Meaningful and relevant issues were elicited and coded into themes in first and second order. With the high volume of raw data obtained from all the transcripts, qualitative analysis software, NVivo was selected and employed for efficient handling, managing, searching, displaying and analyzing of findings. Extract of quotations from senior managers are provided as evidence in the following analysis and discussion.

\section{Findings of Study}

This study reveals that information environment of senior managers is becoming highly uncertain due to over-abundance of information, heterogeneity of information attributes and ambiguous value of information. Senior managers are now suffering from the over-abundance of information. They receive both internal and external information, and from both informal and formal sources. One senior manager complained that, "... to a stage where we were producing far too much information for us to be able to know ... I receive a six pages document on every Monday plus about two or three pages of document for each product type" (Trading Director). Another senior manager gave an example of being overloaded with information, "... because one of the challenges today is when information comes back and you pull it out of the system there tends to be too much information and it's not in the right context" (Chief Financial Officer). The duplication of information sources also causes the excess of information. One manager commented, "There is a fundamental question. Now the question is that we have too much information ... we have newspaper, we have the web, we have the mobile phone, we have all those things in place. How do you process it?" (Managing Director). With the increasing amount of ubiquitous information and heterogeneous information sources, senior managers are pressurized to spend more time and effort to cope with information processing.

In addition to the vast amount of information and the duplication of information sources, senior managers emphasize that the amount of information sources and attributes they received is becoming a problem rather than a benefit to them. Senior managers expressed the different sources and attributes of information they could possibly cope. A senior manager expressed that, "It comes from very diverse sources and format, so it's very time consuming to process them" (Strategic Planning Manager). There is also the challenge of responding to soft information, that is based on opinion or rumor rather than fact. One senior manager raised this concern, "I fear that it will give me too much information, making things more difficult, slowing me down to process; and may be more opinions, more views, making it more difficult to synthesize or summarize and turn it into something that you can act upon" (Head of Chief Executive Officer). Besides the variety sources and attributes of information, senior managers' information environment is diverse and dynamic, influenced by individual and contextual situations. One senior manager gave this example, "I mean I have a range of news sources coming to me daily. I will open them, scan what is in the headlines. If it has the headline terms that are relevant to the topic of my consideration, then I will look deeper. This is done very randomly. It is based on my needs (Chief Executive Officer).

As the business environment continues to produce vast amount of heterogeneous information, senior managers are skeptical about the value of information 
they receive. The underlying problem is that the value of information cannot be assessed until the information arrives. Unexpected and unpopular information sources and attributes can still potentially generate something impactful and valuable for senior managers. One senior manager confirmed by saying, "Even in our business, the most unexpected news has an impact" (Chief Executive Officer).

A highly uncertain information environment of existing senior managers has led to uncertainty in their existing information behavior. Information behavior of senior managers is governed by various concerned issues, specific information needs and personal motivation. Each individual senior manager will scan or probe for information on different occasions, with different issues in mind, and based on different criteria. One senior manager confirmed a personalized nature of information behavior, "The way of choosing information to be examined further, I think it depends on issues, on particular strategic issues where you come across particular piece of information" (Chief Executive Officer).

Senior managers often have time constraint issues with frequent interruptions yet are concerned with a wide range of internal and external business environment issues. The over-abundance and duplication of information sources demand senior managers to spend significant time and effort to process them. As a result, senior managers are very concerned of the time required for information processing. One senior manager argued, "I tend to discard information very quickly. And also I tend not to look at things unless they are interesting." (Chief Executive Officer). The sheer volume of information also suggests the need to reduce information amount and sources, at the same time screen out irrelevant data. One senior manager commented, "You got to dispense irrelevant information very quickly and get to the core and relevant information very quickly" (Chief Executive Officer). Therefore, senior manager's information behavior is considered highly heterogeneous yet personalized and subject to time constraints, which could lead to information avoidance.

\section{Factors Influencing and Shaping Senior Managers' Information Behavior}

It is virtually not feasible to identify explicit patterns of senior managers' information behavior due to the brevity, variety and discontinuity nature of managerial works, as well as uncertainty in managerial information environment and information behavior. However, factors that influence and shape senior managers' information behavior are more likely to be identified. Understanding these factors could provide implications for improving and supporting the informational roles of senior managers as the "nerve center" for the creation, collection and dissemination of information.

Using Thematic Qualitative Analysis (TQA) approach, first order and second order themes were identified and elicited from the raw data (texts). The emerging themes suggest that senior managers' information behavior is influenced and driven by several factors: process-oriented, cognitive-oriented, affective-oriented, situational-oriented, people-driven, technology-driven and strategy-driven. Table 1 depicts the percentages of nodes coded from the overall nodes related to factors influencing and shaping senior managers' information behavior. The percentages are not the main mode of qualitative analysis, but to provide the overview insights of those factors. The results show that information behavior of senior managers is largely influenced and shaped by people who work with them (organizational actors, $\approx 24 \%$ ), followed by their managerial work and organizational contexts (organizational situations, $\approx 20 \%$ ) and moderately driven by feelings (affective responses, $\approx$ $16 \%$ ) and information systems (technological factors, $\approx$ $14 \%$ ). It is, however, less driven and shaped by the thinking efforts of problems solving (cognitive gaps, $\approx$ $9 \%$ ), the systematic stages of information search and process (organizational processes, $\approx 8 \%$ ) and the organizational strategy (organizational strategies, $\approx$ $8 \%)$.

\begin{tabular}{|l|l|}
\hline \multicolumn{1}{|c|}{ Factors } & Percent \\
\hline Organizational actors & $\approx 24 \%$ \\
\hline Organizational situations & $\approx 20 \%$ \\
\hline Affective responses & $\approx 16 \%$ \\
\hline Technological tools & $\approx 14 \%$ \\
\hline Cognitive gaps & $\approx 9 \%$ \\
\hline Process-oriented & $\approx 8 \%$ \\
\hline Strategy-driven & $\approx 8 \%$ \\
\hline Others & $\approx 1 \%$ \\
\hline
\end{tabular}

Table 1. Factors influencing and shaping senior managers' information behavior

\subsection{Organizational Actors}

Although information behavior is inherently individual, the findings show that it is strongly influenced by social factors and sometimes strongly collaborative [2]. The findings reveal that senior managers rely heavily on the people around them in search of trouble signals, messages and missing pieces of puzzles. The findings also show that senior 
managers tend to rely on a specialized organizational unit and team to support their information searching, scanning, refining and reporting process, as illustrated by some of the senior managers below.

"I don't prepare that information, there is a separate unit ... as senior manager, you don't get into information searching and scanning about customer and competitors ... that's already got a whole unit established here and I used that unit to provide information." (Head of Corporate Governance)

Specialized organizational actors known as "knowledge workers" or "information workers" are set up to support senior managers' information processing activities. They are delegated with responsibilities to collect, analyze, prepare and report information that is potentially useful for senior managers.

"We have a business development unit and there we have 5 research people, accumulating all kinds of market and competitor information, analyzing that and putting it into a format, and therefore it is much more structured." (Chief Finance Officer)

In some cases, senior managers would personally request information from the "information workers" for further information process. In other cases, the "information workers" would raise any potentially significant information for senior managers' attention.

"Personally I would access that information via the business planning team. So we have a team who are responsible for business planning and what we call capital allocation, who produce information about how the market is evolving. " (Chief Operating Officer)

The findings imply that collaborative information processing is crucial for senior managers, in which they rely on individual actors to seek, acquire and process information for them. Hwang et. al. (2013)'s findings provide important insights on understanding and improving knowledge workers for better information management practices [9]. In most cases, organizations consist of specialized organizational actors or units that are responsible for supporting and enhancing the process of information gathering, scanning, refining and reporting. Within these organizational units, there are "information workers" or "knowledge workers", who process and provide value-added information for senior managers. Senior managers will then either perform higher level of information process or make decision based on the provision of the value-added information.

\subsection{Organizational Situations}

The findings also suggest that senior managers' information behavior is largely influenced by their work situations and organizational contexts. The value of information is based not only on the subject area or how well the information content matches a particular keyword, query, or topic, but also on the requirements, norms, and conditions that are dependent on the user's work and organizational contexts [6]. Taylor (1991) calls these organizational contexts as information use environments (IUEs) [23]. IUEs are grouped into four categories: sets of people (the nature of their work), problem dimensions (typical concerned problems), work settings (organizational culture, style and structure) and problems resolution assumptions (perceived and anticipated ways to problem resolution).

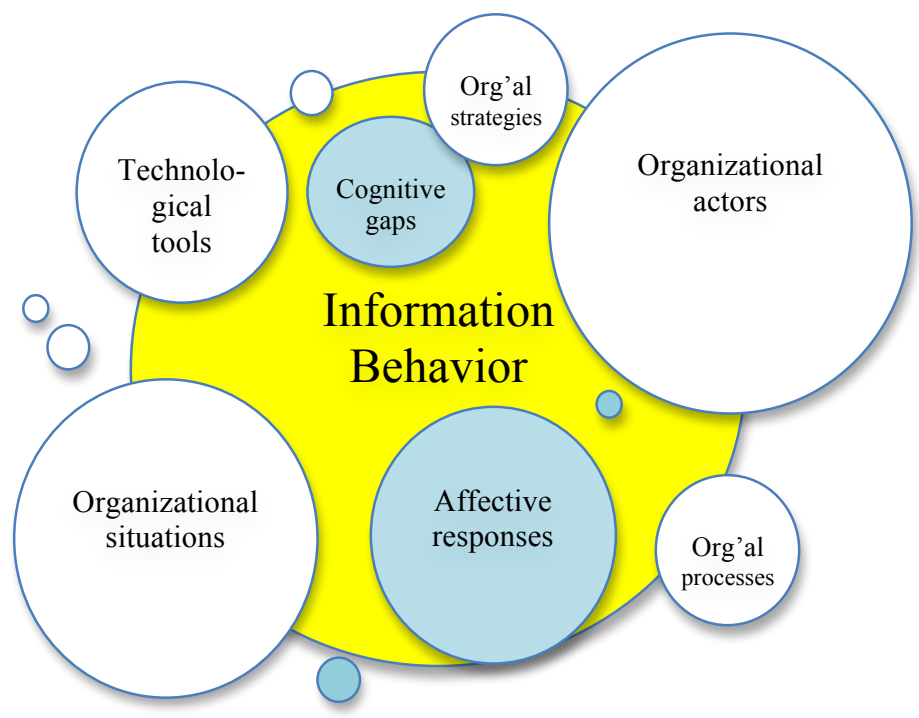

Figures 2. Factors influencing and shaping senior managers' information behavior

In terms of sets of people, the findings confirm that senior managers' educational and professional training backgrounds shape their assumptions and attitudes about the nature of their work that act on their information behavior. For a senior manager who knows his nature of work well, he would find it easier or quicker in his information search and process. One senior manager described the following:

"It's not difficult to assess whether a piece of information is important or not. Because as a senior manager, you know your strategy, your know your neighborhood, you know your organization." (Managing Director)

Problem dimensions refer to the characteristics of the typical problems that a set of people are concerned with [23]. The findings show that senior managers process information based on particular issues (problems) that they are concerned with. However, senior managers are often concerned with dynamic and changing particular issues as new information arrives. This will cause senior managers to change their 
information search and process behavior, as clearly stated by some senior managers:

"It really depends on what we focus on that time, the focus of our business changes from time to time. Sometimes there are things that for one minute they become of interest for specific topics." (Head of IT Strategy)

Work settings are the social and physical attributes of the organization or unit that a set of people work in, in which these attributes influence attitudes toward information needs and information process [6, 7, 23]. The interview confirms some of these attributes that influence senior managers' information behavior, for example, the organization culture and perceived accessibility of information. In particular, one senior manager recognizes the challenge of how organization's working culture would shape people's perceptions about the value of information, hence, influence the information behavior.

"... because what you tend to find is people don't like to tell bad news. Therefore, they would filter the bad news or they would condition somewhere ...because of what people provide and because of the culture as well, we hope the culture isn't wrong here, you could end up with the wrong output based on the right input." (Managing Director)

Problems resolution assumptions are the perceptions shared by a set of people about what constitutes the resolution of their typical issues (problems) [6, 23]. These assumptions guide information search and process, for instance, provide a frame of reference to view and structure the issues, and create expectations about the information traits required to manage or resolve the issues (problems). The findings confirm that senior managers' information behavior is shaped by their perceptions of what information constitutes the resolution of an issue or a problem.

"I will tend to respond on emails that come to me, something that requires my action, or if the information is potentially important that I might need to respond or forward it on." (Managing Director)

In summary, the findings above indicate that senior managers' information behavior is situational-driven, wherein is influenced by the nature of their work, the typical concerned problems, their work settings and their perceptions about problem resolution. The heterogeneity and dynamics of work situations and organizational contexts result in different approaches and intensity of their information search and process.

\subsection{Affective Responses}

The emotional (affective) responses often regulate information processing by channeling attention to potentially important and relevant information, pointing out doubt and uncertainty, indicating likes and dislikes, and motivating effort [6]. The findings show that senior managers' information behavior is often governed by the affective feelings and emotions, rather than the logical and systematic process. The uncertainty of senior managers' information environment drives them to use the affective approach in information seeking and processing. Kuhlthau (1993) describes how the affective responses could ease the uncertainty and increase confidence in the course of information search [13]. One senior manager pointed out:

"I can personally felt that first thing in the morning, I will look at the sales report from the previous day to show top lined sales. I will go through sales first thing in the morning and if it don't feel quite right, I will go into the system to have a look into the individual sales." (Trading Director)

Besides the gut feelings, senior managers also rely on their affective judgment derived from their experiences to make sense of information.

"Judgment, which is based on our experiences. Our managers here in this business, a lot of managers tend to have 10 to 15 years of experience at least. There's no systematic decision making process of using structured information." (Chief Operating Officer)

The findings reveal that the affective feelings and judgment are associated with the past experiences that the senior managers have gained over a period of time. A more experienced senior manager would have better feel and judgment on his information process. These experiences can be industry-related or non-industryrelated. Similarly, Bhattacharjee and Moreno (2002)'s study suggests that professional experience is one factor that influences individuals' assessments of the informational value of affective reactions [3].

"But up to a point, you really have to be based on experience which is fundamentally guts feeling that would come from industry experience, or from experience of other type of industry." (Vice President)

However, senior managers' information behavior is not entirely affective-oriented. In some cases, the information search and process combines both systematic and affective approach. As the information search and process progress, the systematic approach helps senior managers to verify their information, while the affective approach helps senior managers to move from uncertainty to increased certainty, thus the feelings shift toward increased confidence and satisfaction.

"It's a mixture but at the end of the day, when logic can only bring you up to a certain point, because human feelings sometimes don't based on logic, but 
based on a lot of influences and changes that happen at that time..." (Vice President)

To sum up, senior managers' information behavior is influenced by affective feelings and judgment through intuition and experience. The uncertainty of information message motivates the use of affective responses in information search and process. These affective responses help to reduce uncertainty and increase confidence, thus increasing the ability to construct meaning or make sense of the information.

\subsection{Technological Tools}

The findings reveal that senior managers' information behavior is moderately driven and influenced by the use and reliance of information technology or information system. Traditional information systems (technology) are unable to help senior managers to seek trigger, speculative and current information, instead they provide largely internal, historical and precise information of an aggregated and reference nature [15]. Emerging intelligent information systems would have the potential to support strategic information processing. Although senior managers tend to use information systems for internal, historical and aggregated information, the findings suggest that current information systems, such as knowledge-based systems, are designed to, hopefully, provide them with external, current, speculative information of a trigger nature.

"...we implant what we called knowledge-based information system that we have collectively on how certain product information flow so that key respective people will be informed, or triggered will be set to inform right people senior management of what is the developing out there." (Vice President)

The findings imply that senior managers rely on information systems more and more in their information search and process, in particular the corporate database or portal. Many companies either build their own corporate database or they subscribe to information intelligence providers, such as customer and competitor intelligence information.

"The corporate database, which is what we use for the competitor information, everyone in the business has access to that and everyone can input to that, ... and it is live” (Deputy Managing Director)

With the advancement of information technology in AI, senior managers' information behavior is undoubtedly shaped by the use and adoption of information systems. Nevertheless, there is need for developing a more advanced strategic information system that is capable of handling current, speculative and trigger information that are of senior managers' concerns. Thus, senior managers can manage illdefined and uncertain issues with strong evidencebased information.

\subsection{Cognitive Gaps, Organizational Processes and Organizational Strategies}

The findings reveal that senior managers' information behavior is less driven and shaped by the thinking efforts of problems solving (cognitive gaps), the systematic stages of information search and organizational processes, and the organizational strategies. The findings indicate that senior managers' information behavior is ad hoc and does not follow particular thinking patterns and systematic stages due to the brevity, variety and discontinuity nature of their works. Cognitive-oriented approach will be considered when senior managers recognize their inability to act or understand a situation because of a lack of information, but senior managers are overloaded with information today. Senior managers are more concerned with current information and typical problems that require their immediate attention and action. Hence, their information behavior is also fairly driven by business strategy. This suggests that senior managers' information behavior tends to be influenced by current issues rather than long term issues.

\section{Implications for Supporting and Improving Senior Managers' Informational Roles}

The study suggests that senior managers with time constraints tend to rely on the people around them or a special organizational unit for information seeking, gathering, scanning, refining, and analyzing support. This special organizational unit is often addressed as information processing unit or intelligence processing unit, that consists of "information workers", "knowledge workers" or "intelligence specialists", who assist senior managers in information search and process. They need to have a wide knowledge of information sources and skills in exploiting and organizing information, coupled with analytical skills in evaluating and interpreting information [9]. This suggests that senior managers' information behavior can be enhanced with appropriate human assistances, for examples, through social information networks and knowledge workers. Knowledge workers will be responsible for processing and presenting the information in a manageable amount through userfriendly and interactive visualizations.

In addition, these human assistances of information processing activities must be an evolutionary process 
rather than a systematic function, whereby the cognitive, affective and societal development of senior managers would be taken into consideration. The study reveals that senior managers' information processing activities is greatly influenced by their managerial works and organizational contexts. Organization's information culture could also shape senior managers' perceptions about the value of information, hence, influence their information behavior. For instance, senior managers might be concerned with typical issues (problems) at particular situation, context and time. However, their concerns may change as the issues (problems) change over time. This will evidently alter senior managers' attitudes towards information needs and information process. Therefore, the human assistance of information processing activities must also be a continuous learning and adapting process that consider the dynamic work situations, organizational contexts and information culture impacting each individual senior manager.

The study indicates that senior managers' information behavior is governed by the affective feelings and judgment through intuition and experience, rather than just the logical and systematic process. Although the systematic approach helps senior managers verify their information, the affective approach helps to reduce uncertainty and increase confidence, thus increase their ability to construct meaning or make sense of the information. The implication of this study suggests that it is important to support affective responses for two reasons. First, senior managers' incremental training and experiences lead them to trust their gut feelings in the information search and process. Second, as the information search progresses, affective responses are able to shift toward increased confidence and expectations. Technically, it may seem impossible to understand and identify user's affective states, but affective computing is a new area of research, primarily in the recognition and synthesis of facial expression and voice inflection [18, 19]. One feasible example to recognize user's affect is by extracting facemarks (emotions) in text and classifying them into some emotional categories [22]. This may support and enhance information search and process, for example, by building user's information profile with affective categorization. Even though the affective support in information processing is still a long way to go, at least organizations should recognize how the affective states influence senior managers' information processing activities.

With the advancement of information systems and the increasing amount of ubiquitous information, the findings suggest that there is need for developing a more advanced or rational strategic information system for three reasons. First, senior managers' information behavior is dynamic, diverse and ill-defined. Second, senior managers depend on information systems more and more in their information search and process due to the integrated and ubiquitous nature of organizational information systems. Third, the emergence of advanced technologies or systems, such as business intelligence or AI, offer potential solutions in intelligent information processing activities. The goal here is to innovate existing strategic (executive) information systems that are capable of handling current, speculative and trigger information that demands senior managers' attention and action. Another goal is to develop a personalized intelligent support system that is capable of managing and customizing information processes for specific contexts based on senior managers' individual roles and preferences, managerial works, problem dimensions, work settings and problems resolution assumptions. As a result, the system is capable of determining the type of assistance a senior manager needs, learning when (and if) to interrupt the senior manager, and how the senior manager wants to be assisted in different contexts.

\section{Conclusion}

This study seeks to explore factors influencing and shaping existing senior managers' information behavior in light of current competitive business environment and ubiquitous information environment. The aim is then to identify value-added approaches and technological solutions for supporting and improving informational roles of senior managers. Although some implications have been provided here, further study could investigate the extent of how current organizations employ value-added approaches and technological solutions in strategic information processing, such as business intelligence, big data analytics, machine learning and business data visualization. The findings show that information behavior of senior managers is largely influenced and shaped by social factors, followed by their situational and organizational factors. The findings also depict that senior managers' information behavior is moderately influenced and shaped by some affective factors, such as gut feelings, and some technological solutions. Their information behavior is, however, less affected and shaped by the cognitive factors and systematic approaches in information search and process, and is less driven by business strategy. Further study could explore the relationships between these factors and their impact on individual ubiquitous decision-making and problem solving as well as organizational effectiveness and innovation. 


\section{Limitations of Study}

The research sample in this study is relatively small and not industry specific although all interview participants are senior managers. This study is, therefore, exploratory and descriptive, and may not lead to concluding insights for the development of a conceptual model of information behavior. The goal of the findings is to shed light on value-added approaches or technological solutions for supporting and improving information roles of senior managers. However, this requires further research on how senior managers perceive and use the emerging ubiquitous and intelligent information systems.

\section{References}

[1] Bartelings, J.A., Goedee, J., Raab, B. and Bijl, R.. 2017. "The nature of orchestrational work," Public Management Review (19:3), pp. 342-360.

[2] Bawden, D., and Robinson, L. 2013. "No Such Thing as Society? On the Individuality of Information Behaviour," Journal of The American Society For Information Science And Technology (64:12), pp. 2587-2590.

[3] Bhattacharjee, S. and Moreno, K. 2002. "The impact of affective information on the professional judgments of more experienced and less experienced auditors," Journal of Behavioural Decision Making (15:4), pp. 361-377.

[4] Brigham, T.J. 2017. "Merging Technology and Emotions: Introduction to Affective Computing," Medical Reference Services Quarterly (36:4), pp. 399-407.

[5] Case, D.O. \& Given, L.M. 2016. Looking for information: a survey of research on information seeking, needs and behaviour (4th ed.). Emerald Group Publishing.

[6] Choo, C.W. 1998. The Knowing Organisation, New York: Oxford University Press.

[7] Choo, C.W. 2013. "Information Culture and Organisational Effectiveness," International Journal of Information Management (33:5), pp. 775-780.

[8] Dervin, B. 1992. "From the mind's eye of the user: The sense-making qualitative-quantitative methodology," in Qualitative Research in Information Management, J.D. Glazier and R. R. Powell (eds.), Libraries Unlimited, Englewood Cliffs, CO., pp. 61-84.

[9] Hwang, Y., Kettinger, W. and Yi, M.Y. 2013. "A study on the motivational aspects of information management practice," International Journal of Information Management (33), pp.177-184.
[10] Ingwersen, P. 1996. "Cognitive perspective of information retrieval interaction: elements of a cognitive IR theory," Journal of Documentation (52), pp. 3-50.

[11] Kelly, G.A. 1963. A theory of personality: the psychology of personal constructs, New York: W.W. Norton.

[12] Kotter, J.P. 1999. "What Effective General Managers Really Do," Harvard Business Review, pp. 146-159.

[13] Kuhlthau, C.C. 1993. "A principle of uncertainty for information seeking," Journal of Documentation (49:4), pp. 339-455.

[14] Mason, J. 2002. Qualitative researching (2nd ed.), London: Sage.

[15] Mintzberg, H. 1973. The Nature of Managerial Work, New York: Harper and Row.

[16] Morse, J.M. 1997. "Perfectly healthy, but dead; the myth of inter-rater reliability," Qualitative Health Research (7:4), pp. $445-447$.

[17] Nicholas, P. and Anderson, P. 2003. "Quality of life, distress and self-esteem: A focus group study of people with chronic bronchitis," British Journal of Health Psychology (8), pp. 251-270.

[18] Picard, R.W. 1997. Affective computing, MIT Press.

[19] Savolainen, R. 2014. "Emotions as Motivators for Information Seeking: A Conceptual Analysis," Library \& Information Science Research (36:1), pp.59-65.

[20] Smith, J.A., Jarman, M. and Osborn, M. 1999. "Doing interpretative phenomenological analysis," in Qualitative Health Psychology Theories and Methods, M. Murray and K. Chamberlain (eds.), London: Sage Publications, pp. 218-240.

[21] Spink, A. 2010. Information Behaviour: An Evolutionary Instinct, Springer.

[22] Tanaka, Y., Takamura, H. and Okumura, M. 2005. 'Extraction and classification of facemarks', in Proceedings of the 10th international conference on Intelligent user interfaces, San Diego, pp. 28-34.

[23] Taylor, R.S. 1991. "Information use environments," in Progress in Communication Sciences, B. Dervin and M. J. Voigt (eds.), Norwood, NJ: Ablex Publishing, pp. 217-255.

[24] Wilson, T.D. 1999. "Models in information behaviour research," Journal of Documentation (55:3), pp. 249-270.

[25] Wilson, T.D. 2016. "A general theory of human information behavior." Information Research (21:4), pp.1-19.

[26] Zhang, P., \& Soergel, D. 2014. "Towards a comprehensive model of cognitive process and mechanisms of individual sensemaking." Journal Of The American Society For Information Science (65:9), pp.1733-1756. 